\title{
A NEW SIMPLE LIE ALGEBRA OF CHARACTERISTIC THREE
}

\author{
MARGUERITE FRANK
}

\begin{abstract}
We define a restricted simple algebra $T$ of dimension 18 over an arbitrary field of characteristic 3 . From a certain property of its Cartan decomposition, we show $T$ to be nonisomorphic to any known algebra of identical dimension.
\end{abstract}

0 . The algebra $T$ furnishes the first instance of a graded simple Lie algebra:

$$
L=L_{-1} \oplus L_{0} \oplus \cdots \oplus L_{n}, \quad\left[L_{i}, L_{j}\right] \subseteq L_{i+j},
$$

in which $L_{0}$ is a solvable algebra of dimension greater than 1 .

Contained in $T$ is a 10-dimensional simple restricted graded algebra $S$, with $S_{i} \subseteq T_{i}$, and $S_{0}$ solvable, whose newness is still an open question. ${ }^{1}$

1. Definition of $T$. Let $F$ be a field of characteristic 3. The algebras $S$ and $T$, alluded to above, are realized as subalgebras of the Witt-Jacobson algebra $W_{3}$ over $F$. This algebra is spanned by derivations: ${ }^{2}$

$$
A=\left(a_{1}, a_{2}, a_{3}\right)=a_{1} \Delta_{1}+a_{2} \Delta_{2}+a_{3} \Delta_{3},
$$

where $a_{i} \in F\left[x_{1}, x_{2}, x_{3}\right]$ with $x_{i}^{3}=0$, and $\Delta_{i}$ denotes the differential operator $\partial / \partial x_{i}$. If $B=\left(b_{1}, b_{2}, b_{3}\right)$, multiplication in $W_{3}$ is given by $[A, B]=C=$ $\left(c_{1}, c_{2}, c_{3}\right)$, where

$$
c_{i}=\sum_{j}\left[\left(\Delta_{j} a_{i}\right) b_{j}-\left(\Delta_{j} b_{i}\right) a_{j}\right] .
$$

The two algebras have nested gradations

$$
\begin{gathered}
S=S_{-1} \oplus S_{0} \oplus S_{1}, \\
T=T_{-1} \oplus T_{0} \oplus T_{1} \oplus T_{2} \oplus T_{3}, \\
{\left[S_{i}, S_{j}\right] \subseteq S_{i+j}, \quad\left[T_{i}, T_{j}\right] \subseteq T_{i+j}, \quad S_{i} \subseteq T_{i},}
\end{gathered}
$$

Received by the editors March 28, 1972.

AMS (MOS) subject classifications (1970). Primary 17B20.

${ }^{1}$ Although R. Wilson has shown $S$ to be nonisomorphic to the classical matrix algebra of type $B_{2}$, the possibility still remains that $S$ is one of the 10-dimensional algebras of [1], [5], or [6].

${ }^{2}$ Cf. [4].

(c) American Mathematical Society 1973 
where the subspaces $S_{i}$ and $T_{i}$ have the following bases over $F$ :

$$
\begin{aligned}
T_{-1}= & S_{-1}=\left\langle\Delta_{1}, \Delta_{2}, \Delta_{3}\right\rangle, \\
& S_{0}=\left\langle A_{1}=\left(x_{1}, x_{2}, x_{3}\right), A_{2}=\left(0, x_{2},-x_{3}\right),\right. \\
& \left.A_{3}=\left(x_{2}, x_{3}, 0\right), A_{4}=\left(0, x_{1},-x_{2}\right)\right\rangle, \\
& S_{1}=\left\langle B_{1}=\left(x_{1} x_{2}, x_{1} x_{3},-x_{2} x_{3}\right), B_{2}=\left(x_{1}^{2}, x_{1} x_{2}, x_{2}^{2}\right),\right. \\
\left.B_{3}=\left(-x_{2}^{2}, x_{2} x_{3}, x_{3}^{2}\right)\right\rangle, & \\
\text { (1.3) } T_{0}= & S_{0} \oplus\left\langle A_{5}=\left(x_{3}, 0,0\right)\right\rangle, \\
T_{1}= & S_{1} \oplus\left\langle B_{4}=\left(x_{1} x_{3}, 0, x_{3}^{2}\right), B_{5}=\left(x_{2} x_{3},-x_{3}^{2}, 0\right),\right. \\
T_{2}= & \left\langle C_{1}=\left(x_{2}^{2} x_{3}-x_{1} x_{3}^{2}, x_{2} x_{3}^{2}, 0\right),\right. \\
& \left.C_{2}=\left(x_{1}^{2} x_{3}-x_{1} x_{2}^{2}, x_{1} x_{2} x_{3}, x_{2}^{2} x_{3}\right), C_{3}=\left(x_{1} x_{2} x_{3},-x_{1} x_{3}^{2}, x_{2} x_{3}^{2}\right)\right\rangle, \\
T_{3}= & \left\langle D_{1}=\left(x_{1} x_{2}^{2} x_{3}+x_{1}^{2} x_{3}^{2}, x_{1} x_{2} x_{3}^{2}, x_{2}^{2} x_{3}^{2}\right)\right\rangle .
\end{aligned}
$$

THEOREM 1.1. The algebras $S$ and T are restricted central simple algebras with a natural gradation such that $S_{0}^{(4)}=T_{0}^{(4)}=0$.

Proof. We verify at once that

$$
\begin{aligned}
{\left[S_{i}, S_{1}\right] } & =S_{i+1} & & (i=0,1), \\
{\left[T_{i}, T_{-1}\right] } & =T_{i-1} & & (i=1,2,3), \\
{\left[T_{0}, T_{3}\right] } & =T_{3} . & &
\end{aligned}
$$

The simplicity of $S$ and $T$ follows at once from (1.4) and the fact that the set of transformations induced in $S_{-1}, T_{-1}$ by multiplication by elements of $S_{0}$ and $T_{0}$, respectively, is irreducible. ${ }^{3}$ Indeed if $\mathfrak{U} \neq 0$ is an ideal of $S$, then for some $0 \leqq r \leqq 2, \mathfrak{A}\left(\mathrm{ad} S_{-1}\right)^{r} \neq 0 \subseteq S_{-1} \cap \mathfrak{A}$, and the irreducible representation of $S_{0} \rightarrow$ Hom $S_{-1}$ then implies that $\mathfrak{A} \supseteq S_{-1}$. But then, by (1.4), $\mathfrak{A} \supseteq S_{0} \oplus S_{1}, \mathfrak{A}=S$, and $S$ is central simple. Similarly if $\mathfrak{A} \neq 0$ is an ideal of $T$, $\mathfrak{A} \supseteq T_{-1}$ and, by (1.4), $\mathfrak{U} \supseteq$ all $T_{i}$, and $T$ is central simple also.

The restrictedness of $S$ and $T$ follows at once from the restrictedness of $S_{0}$ and $T_{0}$, respectively. ${ }^{4}$ Indeed, denoting by $A^{3}$ in $W_{3}$ the third iterate of the derivation $A$, it is easily verified that $A_{1}^{3}=A_{1}, A_{2}^{3}=A_{2}$, while $A^{3}=0$ for all remaining basis elements of $S$ and $T$.

We finally observe that the derived algebras of $S_{0}$ and $T_{0}$ have the following bases over $F$ :

$$
\begin{array}{lll}
S_{0}^{(2)}=\left\langle A_{1}, A_{3}, A_{4}\right\rangle, & S_{0}^{(3)}=\left\langle A_{1}\right\rangle, & S_{0}^{(4)}=0 . \\
T_{0}^{(2)}=\left\langle A_{1}, A_{3}, A_{4}, A_{5}\right\rangle, & T_{0}^{(3)}=\left\langle A_{1}, A_{3}\right\rangle, & T_{0}^{(4)}=0 .
\end{array}
$$

${ }^{3}$ Theorem 4.3 of [3] states that a naturally graded subalgebra $G$ of the Witt-Jacobson algebra $W_{n}$ containing all $\partial / \partial x_{i}$ is simple if and only if $G=G^{2}, G_{0}=\left[G_{-1}, G_{1}\right], G_{1}=$ $\left[G_{1}, G_{0}\right]$ and the representation of $G_{0}$ in $G_{-1}$ is irreducible.

${ }^{4} \mathrm{Cf}$. Theorem 3.3 of [3]. 
2. Cartan decomposition. The subspace $H=\left\langle A_{1}, A_{2}\right\rangle$ is an abelian subalgebra of $S$ and $T$. For $w \in H^{*}$, define

$$
\begin{aligned}
& T_{w}=\{t \in T \mid t \operatorname{ad}(A)=w(A) t \text { for all } A \in H\}, \\
& S_{w}=\{s \in S \mid s \operatorname{ad}(A)=w(A) s \text { for all } A \in H\} .
\end{aligned}
$$

If $w_{i}\left(A_{j}\right)=\delta_{i j}(i, j=1,2)$, it follows directly that

$$
\begin{array}{rlrl}
H=T_{0} & =\left\langle A_{1}, A_{2}\right\rangle, & \\
T_{w_{1}}=\left\langle B_{2}, B_{5}\right\rangle, & T_{-w_{1}}=\left\langle\Delta_{1}, C_{3}\right\rangle, \\
T_{w_{2}}=\left\langle A_{3}, D_{1}\right\rangle, & T_{-w_{2}}=\left\langle A_{4}, A_{5}\right\rangle, \\
T_{w_{1}+w_{2}}=\left\langle B_{1}, B_{6}\right\rangle, & T_{-w_{1}-w_{2}}=\left\langle\Delta_{2}, C_{2}\right\rangle, \\
T_{w_{1}-w_{2}}=\left\langle B_{3}, B_{4}\right\rangle, & T_{-w_{1}+w_{2}}=\left\langle\Delta_{3}, C_{1}\right\rangle . \\
H=S_{0} & =\left\langle A_{1}, A_{2}\right\rangle, & \\
S_{w_{1}} & =\left\langle B_{2}\right\rangle, & & \\
S_{w_{2}} & =\left\langle A_{3}\right\rangle, & S_{-w_{1}} & =\left\langle\Delta_{1}\right\rangle, \\
S_{w_{1}+w_{2}} & =\left\langle B_{1}\right\rangle, & S_{-w_{2}} & =\left\langle A_{4}\right\rangle, \\
S_{w_{1}-w_{2}} & =\left\langle B_{3}\right\rangle, & S_{-w_{1}-w_{2}} & =\left\langle\Delta_{2}\right\rangle,
\end{array}
$$

Thus $H$ is a splitting Cartan subalgebra of both $S$ and $T$, with roots $\alpha=\lambda_{1} w_{1}+\lambda_{2} w_{2}$ for integers $\lambda_{i}=-1,0,1$.

3. Newness of $T$. The only known simple algebra of dimension 18 is the Witt-Jabobson algebra $W_{2}$. As shown in [2], every Cartan subalgebra of $W_{2}$ is conjugate to one and only one of

$$
\begin{gathered}
H_{1}=\left\langle\left(x_{1}, 0\right),\left(0, x_{2}\right)\right\rangle, \quad H_{2}=\left\langle\left(x_{1}+1,0\right),\left(0, x_{2}\right)\right\rangle, \\
H_{3}=\left\langle\left(x_{1}+1,0\right),\left(0, x_{2}+1\right)\right\rangle .
\end{gathered}
$$

If $H$ is a Cartan subalgebra of a Lie algebra $L$, let $n(L, H)$ denote the number of pairs (unordered) of roots $\{\alpha,-\alpha\}$ such that $\left[L_{\alpha}, L_{-\alpha}\right]=H$. Then $n(L, H)$ depends only on the conjugacy of $H$. We prove ${ }^{5}$

LEMMA 3.1. If $H$ is a Cartan subalgebra of $W_{2}$, then $n\left(W_{2}, H\right) \geqq 2$.

Proof. By writing $H=\left\langle\theta_{1}=\left(y_{1}, 0\right), \theta_{2}=\left(0, y_{2}\right)\right\rangle$, where $y_{1}=x_{1}$ or $x_{1}+1$, $y_{2}=x_{2}$ or $x_{2}+1$, we can prove the lemma for all three $H_{i}$ at once. Let

$$
U_{w}=\left\{u \in W_{2} \mid u \operatorname{ad}(\theta)=w(\theta) u \text { for all } \theta \in H\right\} .
$$

\footnotetext{
${ }^{5}$ The author is indebted to R. Wilson for suggesting a proof based on [2] much simpler than her original one. The related proof given here is even shorter.
} 
Letting $w_{i}\left(\theta_{j}\right)=\delta_{i j}$ for $i, j=1,2$, we determine

$$
\begin{array}{ll}
U_{w_{1}}=\left\langle\left(y_{1}^{2}, 0\right),\left(0, y_{1} y_{2}\right)\right\rangle, & U_{-w_{1}}=\left\langle(1,0),\left(0, y_{1}^{2} y_{2}\right)\right\rangle, \\
U_{w_{2}}=\left\langle\left(y_{1} y_{2}, 0\right),\left(0, y_{2}^{2}\right)\right\rangle, & U_{-w_{2}}=\left\langle\left(y_{1} y_{2}^{2}, 0\right),(0,1)\right\rangle .
\end{array}
$$

It is at once immediate that $\left[U_{w_{1}}, U_{-w_{1}}\right]=\left[U_{w_{2}}, U_{-w_{2}}\right]=H$ for all allowable substitutions for $y_{1}$ and $y_{2}$. Thus $n\left(W_{2}, H\right) \geqq 2$.

THEOREM 3.1. The algebra $T$ is not isomorphic to $W_{2}$ and is therefore new.

Proof. For $\alpha=w_{1}, w_{2}, w_{1}+w_{2}$ the subspace $\left[T_{\alpha}, T_{-\alpha}\right]$ is equal to $\left\langle A_{1}+A_{2}\right\rangle,\left\langle A_{1}\right\rangle,\left\langle A_{1}-A_{2}\right\rangle$, respectively. While $\left[T_{w_{1}-w_{2}}, T_{-w_{1}+w_{2}}\right]=H$. Hence $n(T, H)=1$, and by Lemma 3.1, $T$ cannot be isomorphic to $W_{2}$.

\section{BIBLIOGRAPHY}

1. G. Brown, Lie algebras of characteristic three with nondegenerate Killing form, Trans. Amer. Math. Soc. 137 (1969), 259-268. MR 39 \#2825.

2. S. P. Demuškin, Cartan subalgebras of the simple Lie algebra $W_{n}$ and $S_{n}$, Sibirsk. Mat. Ż. 11 (1970), 310-325=Siberian Math. J. 11 (1970), 233-245. MR 41 \#6919.

3. M. Frank, On a theory relating matric Lie algebras of characteristic $p$ and subalgebras of the Witt-Jacobson algebra, Progress Report I.T., Math. Dept., University of Minnesota, Minneapolis, Minn., 1943, pp. 107-121.

4. N. Jacobson, Classes of restricted Lie algebras of characteristic p. II, Duke Math. J. 10 (1943), 107-121. MR 4, 187.

5. I. Kaplansky, Lie algebras of characteristic p, Trans. Amer. Math. Soc. 89 (1958), 149-183. MR 20 \#5799.

6. A. I. Kostrikin, A parametric family of simple Lie aigebras, Izv. Akad. Nauk SSSR Ser. Mat. 34 (1970), 744-756=Math. USSR Izv. 4 (1970), 751-764. MR 43 \#302.

115 Broadmead, Princeton, New Jersey 08540 